\title{
3D MODELLING OF DISTRIBUTION OF TEMPERATURE FIELD IN THE ROLLING MILL
}

\author{
Yedilkhan Amirgaliyev', Waldemar Wójcik² ${ }^{\text {, Tleugaisha Ospanova }}{ }^{3}$, Kabilda Jetpisov ${ }^{3}$ \\ 1 Institute of Information and Computational Technologies, Almaty, Kazakhstan, e-mail: amir_ed@mail.ru \\ 2 Lublin University of Technology, Nadbystrzycka 38d, 20-618 Lublin, Poland, e-mail: waldemar.wojcik@pollub.pl \\ ${ }^{3}$ Eurasian National University named after L.N. Gumilyev, Astana, Kazakhstan, e-mail: tleu2009@mail.ru, \\ Jetpisov_K54@mail.ru
}

Received: 2017.08.15

Accepted: 2017.10.01

Published: 2017.11.01

\begin{abstract}
This article is dedicated to the study of numerical simulation of temperature field distribution in rolling mill with many cages for continuous casting and rolling stripe of metal wire. In work the simplest is used in use, certainly a steady, economic difference method, a method of division of variables of a multidimensional task into a chain of one-dimensional tasks, a method of total approximation, the locally one-dimensional scheme. The algorithm of numerical modeling of distribution of a temperature field on cages of the rolling mill is constructed. Solutions of the threedimensional temperature field distribution tasks based on experimental data allows finding the temperature across the strip of metal rod in a rolling mill stands. Calculation of the distribution of temperature field in hearth of deformation of a metal rod was realized on implicit scheme. The calculations are shown in the graphs. It provides an analysis of the error of errors.
\end{abstract}

Keywords: cage, hearth of deformation, rolling, temperature field, the locally one-dimensional scheme

\section{INTRODUCTION}

In the conditions of processing of metals pressure among the factors influencing plasticity as structure and structure of deformable metal, character of a tension at deformation, deformation speed, etc. is deformation temperature. Question of modeling of temperature fields, the describing temperature processes happening in the center of deformation and a technological stream of a camp were engaged many scientists. For creation of this algorithm the model [Dubinsky and Sosedkova 2007] was used. In work [Sosedkova et al. 2010] this model is realized by means of a method of final elements.

At the numerical solution of problems of distribution of a temperature field on cages of the closed rolling mill for the line of continuous molding and rolling of a metal rod one of the most common method is the method of grids. Simplicity and universality are characteristic for difference methods for solving boundary value problems of mathematical physics in the regular settlement areas, and the use of irregular grids making closer the finite difference method with the finite element method. In work has been used economical the difference method for the solution of multidimensional equations in private derivatives [Samarskyi 1989], the method of total approximation, which allows you to get absolutely stable converging locally - one-dimensional schemes for the equations of parabolic type.

\section{RESEARCH OBJECTIVE}

For numerical modeling of a temperature field on cages of the rolling mill we will con- 
sider a task for the heat conductivity equation in the form of [Sosedkova et al. 2010]:

$$
c \rho \frac{\partial U}{\partial t}=\operatorname{div}(\lambda \cdot \operatorname{grad} U)+\tau_{s} H
$$

where: $c$-specific heat of metal;

$\rho$ - mass density of metal;

$\lambda$ - thermal conductivity coefficient;

$\tau_{s}$ - resistance of metal of plastic deformation of shift;

$H$ - intensity of speeds of deformation of shift, with the following boundary conditions:

At the entrance of the deformation center, the temperature of the metal known;

On a contact surface takes place the heat exchange between deformable metal and a surface of rolls described by expression:

$$
\lambda \frac{\partial U}{\partial n}=\alpha_{s}\left(U-U_{b}\right)
$$

where: $\alpha_{\mathrm{s}}-$ heat transfer coefficient on a contact surface;

$U_{b}-$ temperature of rolls.

On other surfaces, limiting the deformation center, the thermal stream is equal to zero.

At the exit from $i-$ oh cages for practical calculations of temperature in multistand rolling mill we will use the simplified model [Dubinsky and Sosedkova 2007] which has an appearance:

$$
U_{i}=U_{i-1}-\Delta U_{u}-\Delta U_{k}-\Delta U_{b}+\Delta U_{d}
$$

where: $U_{i}-$ temperature at the exit from $i-$ oh cages,

$U_{i-1}-$ temperature at the exit from the previous cage or temperature of initial heating of preparation,

$\Delta U_{\dot{e}}$ - a heat transfer radiation in environment which is described by StephaneBoltsman's formula,

$\Delta U_{k}-$ a convective heat transfer,

$\Delta U_{b}$ - contact heat exchange with working rolls,

$\Delta U_{d}$ - a metal warming up due to energy of plastic deformation.

According to the theory in the majority of processing of metal the form of preparation differs in pressure from the form of a finished product determined by a tool form. The closer the element is to a section corner, the he will receive smaller lengthening. Therefore the parties of section will receive a convex form. Square section will come nearer to circular, and rectangular - at first to an ellipse, and then all the same to a circle. For experiment at a choice of a form of calibers of cages rectangular section was taken.

\section{Difference equation}

For drawing up algorithm of the numerical solution of the formulated task we will consider the three-dimensional parabolic equation of the second order in a general view [Samarskyi 1989]:

$$
\begin{gathered}
\frac{\partial U}{\partial t}=\sum_{\alpha=1}^{3} L_{\alpha} U+f(x, t) \\
L_{\alpha} U=\frac{\partial}{\partial x_{\alpha}}\left(k_{\alpha}(x, t) \frac{\partial U}{\partial x_{\alpha}}\right), \\
k_{\alpha}(x, t) \geq c_{1}>0, c_{1}=\text { const },
\end{gathered}
$$

where: $x=\left(x_{1}, x_{2}, x_{3}\right)$ - point of 3-dimensional space with coordinates $X_{1}, X_{2}, X_{3}$. Let $\mathrm{G}$ - any-dimensional 3 - area with border of $\Gamma, \overline{\mathrm{G}}=\mathrm{G}+\Gamma$,

$$
\begin{aligned}
& \bar{Q}_{T}=\bar{G} \times[0 \leq t \leq T], \\
& Q_{T}=G \times(0<t \leq T] .
\end{aligned}
$$

Is to find a continuous cylinder $\bar{Q}_{T}$ solution of the equation (4), meeting a regional condition

$$
U=\mu(x, t) \text { at } x \in \Gamma, 0 \leq t \leq T,
$$

and the initial condition:

$$
U(x, 0)=U_{0}(x) \text {, at } x \in \bar{G} .
$$

As usual, it is supposed that this task has the only decision $U=U(x, t)$, possessing all derivatives demanded on the statement course.

We pass to writing of the local and one-dimensional scheme now. We will construct a uniform grid $\omega_{h r}$ :

$\omega_{h}=\left\{\begin{array}{l}x_{\alpha}=i_{\alpha} \cdot h_{\alpha}, \quad i_{\alpha}=\overline{0, N_{\alpha}}, N_{\alpha}=\frac{l_{\alpha}}{h_{\alpha}}, \quad \alpha=1,2,3 \\ t(j)=j \cdot \tau, j=\overline{0, m}, \quad m=\frac{m k}{\tau}\end{array}\right\}$

When building a locally one-dimensional scheme formally replace the three-dimensional equation of one-dimensional chain of equations, ie, approximate increments $\frac{\tau}{3}$ consistently
operators: 


$$
\mathfrak{R}_{\alpha} U=\frac{1}{3} \frac{\partial U}{\partial t}-\left(L_{\alpha} U+f_{\alpha}\right), \alpha=1,2,3
$$

where $f_{\alpha}$ meets a condition $\sum_{\alpha=1}^{3} f_{\alpha}=f$.

For approximation $L_{\alpha} U+f_{\alpha}$ on a spatial grid $\omega_{h}$ we will use the uniform differential operator of the second order of approximation $\Lambda_{\alpha} y+\varphi_{\alpha}$. Boundary conditions and right part $\varphi_{\alpha}$ undertake in any time points:

$$
\varphi_{\alpha}^{j+\frac{\alpha}{3}}=f_{\alpha}\left(x, t_{j+0.5}\right), \quad \mu^{j+\frac{\alpha}{3}}=\mu\left(x, t_{j+\alpha / 3}\right), \quad \alpha=1,2,3 .
$$

Approximating each heat equation numbers $\alpha$ on the interval $t_{j+(\alpha-1) / 3}<t \leq t_{j+a / 3}$ a twolayer scheme with weights, we obtain a chain $p$ dimensional scheme which called locally onedimensional scheme:

$$
\begin{aligned}
& \frac{U^{j+\frac{\alpha}{3}}-U^{j+\frac{\alpha-1}{3}}}{\tau}=\Lambda_{\alpha}\left(\sigma U^{j+\frac{\alpha}{3}}+(1-\sigma) U^{j+\frac{\alpha-1}{3}}\right)+\varphi_{\alpha}^{j+\frac{\alpha}{3}}, \\
& \alpha=1,2,3, \quad x \in \omega_{h}
\end{aligned}
$$

where: $\sigma$-arbitrary number

Consider a purely implicit locally one-dimensional scheme, at $\sigma=1$ :

$$
\begin{aligned}
& \frac{y^{j+\frac{\alpha}{3}}-y^{j+\frac{\alpha-1}{3}}}{\tau}=\Lambda_{\alpha} y^{j+\frac{\alpha}{3}}+\varphi_{\alpha}^{j+\frac{\alpha}{3}}, \\
& \alpha=1,2,3, \quad x \in \omega_{h}
\end{aligned}
$$

The boundary condition:

$$
\begin{aligned}
& y^{j+\frac{\alpha}{3}}=\mu^{j+\frac{\alpha}{3}} \text { at } x \in \gamma_{h, \alpha}, \\
& j=0,1, \ldots, j_{0}, \alpha=1,2,3
\end{aligned}
$$

The initial condition:

$$
y(x, 0)=u_{0}(x)
$$

Let us more detail on (9):

$$
\begin{aligned}
& \frac{y^{j+\frac{1}{3}}-y^{j}}{\tau}=\Lambda_{1}\left(y^{j+\frac{1}{3}}\right)+f_{1}^{j+\frac{1}{3}} \\
& \frac{y^{j+\frac{2}{3}}-y^{j+\frac{1}{3}}}{\tau}=\Lambda_{2}\left(y^{j+\frac{2}{3}}\right)+f_{2}^{j+\frac{2}{3}}, j=\overline{1, m-1} \\
& \frac{y^{j+1}-y^{j+\frac{2}{3}}}{\tau}=\Lambda_{3}\left(y^{j+1}\right)+f_{3}^{j+1}
\end{aligned}
$$

The boundary condition:

$$
\begin{gathered}
\left.y^{j+\frac{\alpha}{3}}\right|_{x_{a}=0}=\left.\mu\left(x, t_{j+\frac{\alpha}{3}}\right)\right|_{x_{a}=0},\left.y^{j+\frac{\alpha}{3}}\right|_{x_{a}=I_{\alpha}}= \\
=\left.\mu\left(x, t_{j+\frac{\alpha}{3}}\right)\right|_{x_{a}=I_{\alpha}}, \alpha=1,2,3
\end{gathered}
$$

The initial condition:

$$
y^{0}=U_{0}(x)
$$

Let us consider each of the three separate equations (12). First of the equation (12) write at the form:

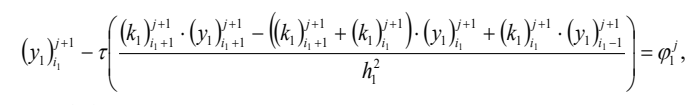$$
\varphi_{1}^{\prime}=\left(y_{3}\right)^{\prime}+\tau \cdot f_{1}^{\prime}
$$

where: $j=\overline{0, m}-1$,

$$
\begin{aligned}
& i_{1}=\overline{1, n_{1}-1}, \\
& i_{2}=\overline{0, n_{2}}, \\
& i_{3}=\overline{0, n_{3}}, \\
& f_{1}^{j}=f\left(x, t_{j}+\frac{\tau}{2}\right), j=\overline{1, m}
\end{aligned}
$$

(15) rewrite at the form:

$$
\begin{aligned}
& \left(1+\frac{\tau}{h_{1}^{2}}\left(\left(k_{1}\right)_{i_{1}+1}^{j+1}+\left(k_{1}\right)_{i_{1}}^{j+1}\right)\right) \cdot\left(y_{1}\right)_{i_{1}}^{j+1}- \\
& -\frac{\tau}{h_{1}^{2}}\left(k_{1}\right)_{i_{1}+1}^{j+1} \cdot\left(y_{1}\right)_{i_{1}+1}^{j+1}-\frac{\tau}{h_{1}^{2}}\left(k_{1}\right)_{i_{1}}^{j+1} \cdot\left(y_{1}\right)_{i_{1}-1}^{j+1}=\varphi_{1}^{j}
\end{aligned}
$$

Denoting:

$$
A_{i_{1}}=\frac{\tau}{h_{1}^{2}}\left(k_{1}\right)_{i_{1}}^{j+1}, C_{i_{1}}=1+\left(A_{i_{1}+1}+A_{i_{1}}\right), F_{i_{1}}=\varphi_{1}^{j}
$$

get:

$$
\begin{aligned}
& A_{i_{1}} \cdot\left(y_{1}\right)_{i_{1}-1}^{j+1}-C_{i_{1}} \cdot\left(y_{1}\right)_{i_{1}}^{j+1}+A_{i_{1}+1} \cdot\left(y_{1}\right)_{i_{1}+1}^{j+1}=-F_{i_{1}}, \\
& i_{1}=\overline{1, n_{1}-1, i_{2}}=\overline{1, n_{2}-1}, i_{3}=1, n_{3}-1
\end{aligned}
$$

Boundary conditions generally:

$$
\begin{aligned}
& \left(y_{1}\right)_{0}^{j+1}=\chi_{1} \cdot\left(y_{1}\right)_{1}^{j+1}+\mu_{11}^{j+1}, \\
& \left(y_{1}\right)_{n_{1}}^{j+1}=\chi_{2} \cdot\left(y_{1}\right)_{n_{1}-1}^{j+1}+\mu_{12}^{j+1}
\end{aligned}
$$

where: $\chi_{1}=0, \chi_{2}=0$ - First type boundary conditions, $\quad \mu_{11}^{j+1}=y^{0}, \mu_{21}^{j+1}=U_{i}$, where $U_{i}-$ the temperature outlet $i-$ in (3).

Next, substituting known $y^{0}$ values are calculated $F_{1}$, and then sweep method solve the problem (17) in all the nodes of the grid $\omega_{h}$ : 


$$
\begin{aligned}
& \alpha_{i_{1}+1}=\frac{A_{i_{1}+1}}{C_{i_{1}}-A_{i_{1}} \cdot \alpha_{i_{1}}}, \alpha_{1}=\chi_{1}, \\
& \beta_{i_{1}+1}=\frac{A_{i_{1}+1} \cdot \beta_{i_{1}}+F_{i_{1}}}{C_{i_{1}}-A_{i_{1}} \cdot \alpha_{i_{1}}}, \beta_{1}=\mu_{11}^{0}, \\
& \left(y_{1}\right)_{n_{1}}^{j+1}=\frac{\mu_{12}^{j+1}+\chi_{2} \cdot \beta_{n_{1}}}{1-\chi_{2} \cdot \alpha_{n_{1}}}, \\
& \left(y_{1}\right)_{i_{1}}^{j+1}=\alpha_{i_{1}+1} \cdot\left(y_{1}\right)_{i_{1}+1}^{j+1}+\beta_{i_{1}+1}, \overline{1, n_{3}-1} \\
& i_{1}=\overline{n_{1}-1,0}, i_{2}=\overline{1, n_{2}-1}, i_{3}=\overline{10}
\end{aligned}
$$

The second of the equation (12) is written in the form:

$\left(y_{2}\right)_{i_{1}}^{j+1}-\tau\left(\frac{\left(k_{2}\right)_{i_{2}+1}^{j+1} \cdot\left(y_{2}\right)_{i_{2}+1}^{j+1}-\left(\left(k_{2}\right)_{i_{2}+1}^{j+1}+\left(k_{2}\right)_{i_{2}}^{j+1}\right) \cdot\left(y_{2}\right)_{i_{2}}^{j+1}+\left(k_{2}\right)_{i_{2}}^{j+1} \cdot\left(y_{2}\right)_{i_{2}-1}^{j+1}}{h_{2}^{2}}\right)=\varphi_{2}^{j}$,

$\varphi_{2}^{j}=\left(y_{1}\right)^{j+1}+\tau \cdot f_{2}^{j}$

where: $j=\overline{0, m}-1$,

$$
\begin{aligned}
& i_{1}=\overline{1, n_{1}-1}, \\
& i_{2}=\overline{0, n_{2}}, \\
& i_{3}=\overline{0, n_{3}}, \\
& f_{2}^{j}=f\left(x, t_{j}+\frac{\tau}{2}\right), j=\overline{1, m}
\end{aligned}
$$

(20) rewrite at the form:

$$
\begin{gathered}
\left(1+\frac{\tau}{h_{2}^{2}}\left(\left(k_{2}\right)_{i_{2}+1}^{j+1}+\left(k_{2}\right)_{i_{2}}^{j+1}\right)\right) . \\
\cdot\left(y_{2}\right)_{i_{2}}^{j+1}-\frac{\tau}{h_{2}^{2}}\left(k_{2}\right)_{i_{2}+1}^{j+1} \cdot \\
\cdot\left(y_{2}\right)_{i_{2}+1}^{j+1}-\frac{\tau}{h_{2}^{2}}\left(k_{2}\right)_{i_{2}}^{j+1} . \\
\cdot\left(y_{2}\right)_{i_{2}-1}^{j+1}=\varphi_{2}^{j}
\end{gathered}
$$

Denoting,

$$
A_{i_{2}}=\frac{\tau}{h_{2}^{2}}\left(k_{2}\right)_{2}^{j+1}, C_{i_{2}}=1+\left(A_{i_{2}+1}+A_{i_{2}}\right), F_{i_{2}}=\varphi_{2}^{j}
$$

get:

$$
\begin{aligned}
& A_{i_{2}} \cdot\left(y_{2}\right)_{i_{2}-1}^{j+1}-C_{i_{2}} \cdot\left(y_{2}\right)_{i_{2}}^{j+1}+A_{i_{2}+1} \cdot \\
& \cdot\left(y_{2}\right)_{i_{2}+1}^{j+1}=-F_{i_{2}}, \\
& i_{1}=\overline{1, n_{1}-1, i_{2}}=\overline{1, n_{2}-1}, i_{3}=\overline{1, n_{3}-1}
\end{aligned}
$$

Boundary conditions generally:

$$
\begin{aligned}
& \left(y_{2}\right)_{0}^{j+1}=\chi_{1} \cdot\left(y_{2}\right)_{1}^{j+1}+\mu_{21}^{j+1} \\
& \left(y_{2}\right)_{n_{2}}^{j+1}=\chi_{2} \cdot\left(y_{2}\right)_{n_{2}-1}^{j+1}+\mu_{22}^{j+1}
\end{aligned}
$$

where:

$$
\chi_{1}=\lambda /\left(\lambda+\alpha_{\beta} \cdot h_{2}\right), \quad \chi_{2}=\lambda /\left(\lambda+\alpha_{\beta} \cdot h_{2}\right)
$$

- third type boundary conditions.

Next, substituting known values $y_{1}^{j+1}$ are $F_{2}$ calculated, and then sweep method solve the problem (22) in all the nodes of the grid $\omega_{h}$. The third of the equation (14) is written in the form:

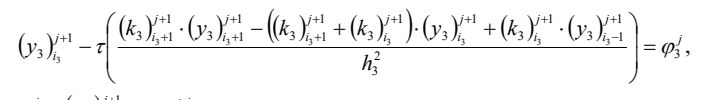

$\varphi_{3}^{\prime}=\left(y_{2}\right)^{i+1}+\tau \cdot f_{3}^{\prime}$

where: $j=\overline{0, m}-1$,

$$
\begin{aligned}
& i_{1}=\overline{1, n_{1}-1}, \\
& i_{2}=\overline{0, n_{2}}, \\
& i_{3}=\overline{0, n_{3}}, \\
& f_{1}^{j}=f\left(x, t_{j}+\frac{\tau}{2}\right), j=\overline{1, m}
\end{aligned}
$$

(24) rewrite at the form:

$$
\begin{aligned}
& \left(1+\frac{\tau}{h_{3}^{2}}\left(\left(k_{3}\right)_{i_{3}+1}^{j+1}+\left(k_{3}\right)_{i_{3}}^{j+1}\right)\right) * \\
& *\left(y_{3}\right)_{i_{3}}^{j+1}-\frac{\tau}{h_{3}^{2}}\left(k_{3}\right)_{i_{3}+1}^{j+1} * \\
& *\left(y_{3}\right)_{i_{3}+1}^{j+1}-\frac{\tau}{h_{3}^{2}}\left(k_{3}\right)_{i_{3}}^{j+1} * \\
& *\left(y_{3}\right)_{i_{3}-1}^{j+1}=\varphi_{3}^{j}
\end{aligned}
$$

Denoting,

$$
A_{i_{3}}=\frac{\tau}{h_{3}^{2}}\left(k_{3}\right)_{i_{3}}^{j+1}, C_{i_{3}}=1+\left(A_{i_{3}+1}+A_{i_{3}}\right), F_{i_{3}}=\varphi_{3}^{j}
$$

get:

$$
\begin{aligned}
& A_{i_{3}} \cdot\left(y_{3}\right)_{i_{3}-1}^{j+1}-C_{i_{3}} \cdot\left(y_{3}\right)_{i_{3}}^{j+1}+A_{i_{3}+1} \cdot \\
& \cdot\left(y_{3}\right)_{i_{3}+1}^{j+1}=-F_{i_{3}}, \\
& i_{1}=\overline{1, n_{1}-1, i_{2}}=\overline{1, n_{2}-1}, i_{3}=\overline{1, n_{3}-1}
\end{aligned}
$$

Boundary conditions generally:

$$
\begin{aligned}
& \left(y_{3}\right)_{0}^{j+1}=\chi_{1} \cdot\left(y_{3}\right)_{1}^{j+1}+\mu_{31}^{j+1}, \\
& \left(y_{3}\right)_{n_{3}}^{j+1}=\chi_{2} \cdot\left(y_{3}\right)_{n_{3}-1}^{j+1}+\mu_{32}^{j+1}
\end{aligned}
$$

where: $\chi_{1}=\lambda /\left(\lambda+\alpha_{\beta} \cdot h_{3}\right)$,

$$
\chi_{2}=\lambda /\left(\lambda+\alpha_{\beta} \cdot h_{3}\right)
$$

Third type boundary conditions.

Next, substituting known values $y_{1}^{j+1}$ are $F_{2}$ calculated, and then sweep method solve the problem (26) in all the nodes of the grid $\omega_{h}$. 
The solution is $\left(y_{3}\right)_{i_{3}}^{j+1}$.

\section{The results of numerical calculations}

In order to test the proposed algorithm is numerically solved the problem (4) in the parallelepiped and the relative errors were obtained by calculations in the corresponding norm [Ryndin 2003]:

$$
\begin{aligned}
& \varepsilon_{i j}=\frac{\max \left|y^{j}-y^{j-1}\right|}{\max \left|y^{0}\right|}, \\
& i=\overline{1,11}, j=1,2, \ldots, j_{0}
\end{aligned}
$$

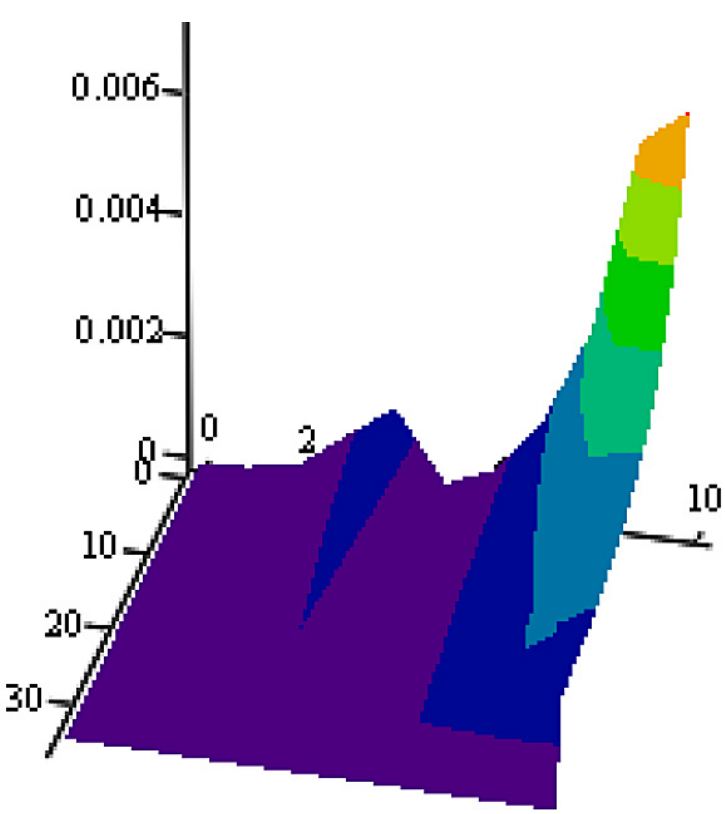

Fig. 1. The results of calculating the relative error

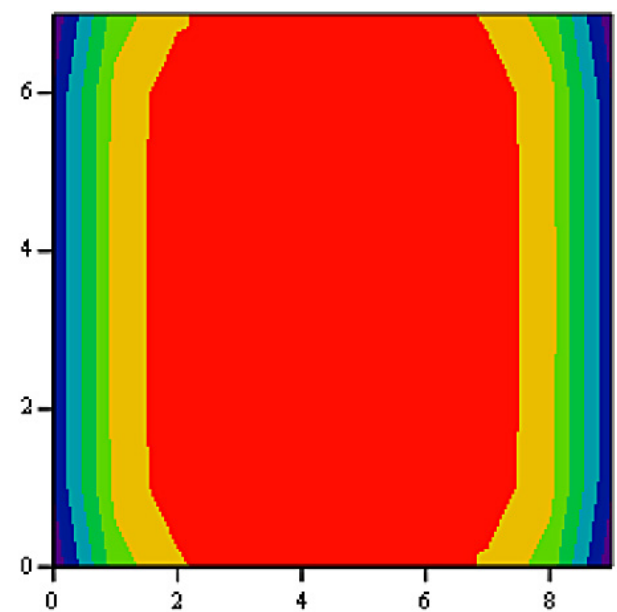

zy5 where: $j$-iteration number,

$$
i-\text { number of stands. }
$$

The results of calculations of the relative error $\varepsilon_{i j}$ shown in Figure 1. When the implementation of the computing process each successive approximation to the solution or rather the previous one, ie, error $\varepsilon_{i j}$ decreases with each iteration. So iterative process converges.

The developed algorithm for solving the problem of temperature conditions allows for temperature changes directly into the rolling stand and the interstand gaps in the rolling mill. The algorithm can be used to calculate the temperature distribution of various rolled materials on any type of profiled rolling mill.

The program implements the algorithm for solving the temperature field distribution of the metal rod on a rolling mill stand is written in Fortran PoverStation 4.0. For the calculation as input data from the literature were used. As a result of the calculation were obtained: three-dimensional temperature field. The results of studies of the temperature field of the metal rod from alloy VT6.

Initially heated to a temperature of $950{ }^{\circ} \mathrm{C}$ the preform is fed into a first roughing mill, after rolling in the stand surface of the strip is cooled to a temperature in the range $904-949^{\circ} \mathrm{C}$. Figure 2 shows a cross-section of roll after the fifth stand, where the surface temperature of roll in contact with the roller, due to heat transfer is reduced to $880^{\circ} \mathrm{C}$, resulting in a greater plastic deformation angle bars and more strain warming seen smaller drop in temperature - up

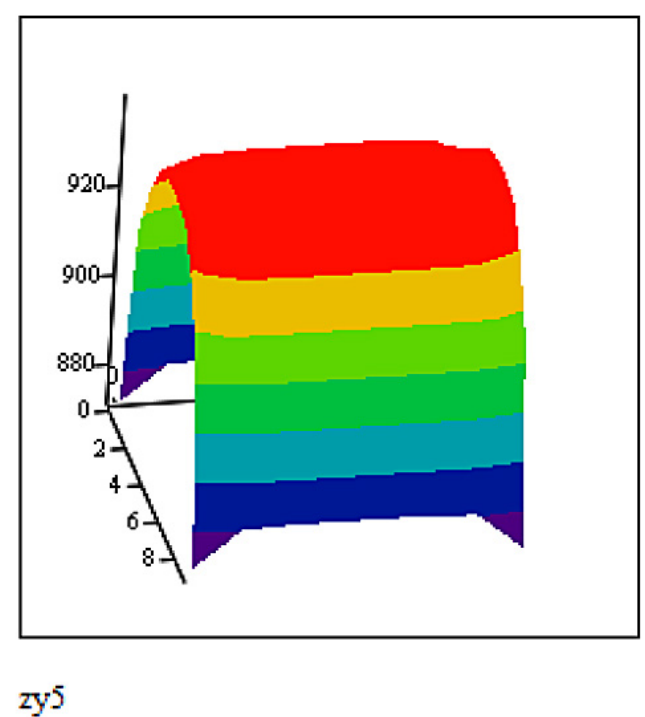

Fig. 2. The cross-section of roll cage after the fifth stand 


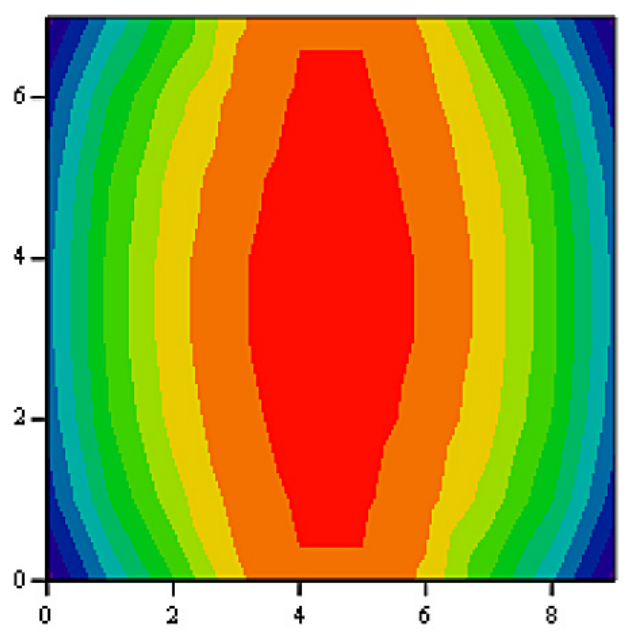

zy11n

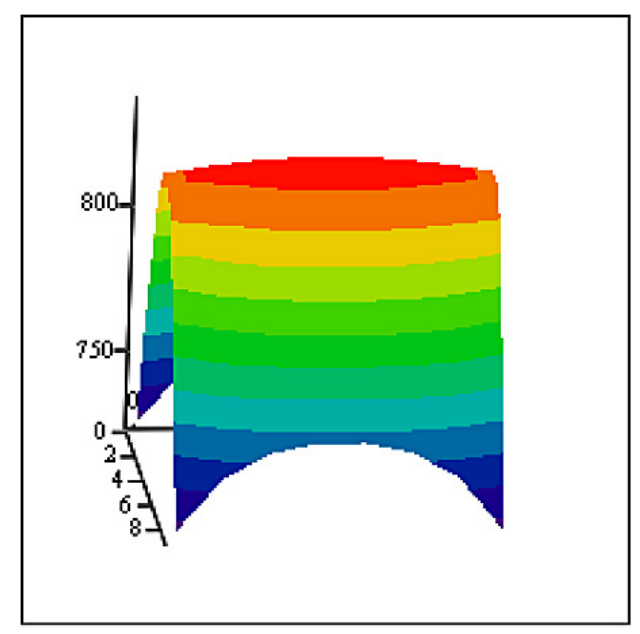

zy11n

Fig. 3. The cross-section of roll cage after the eleventh passages

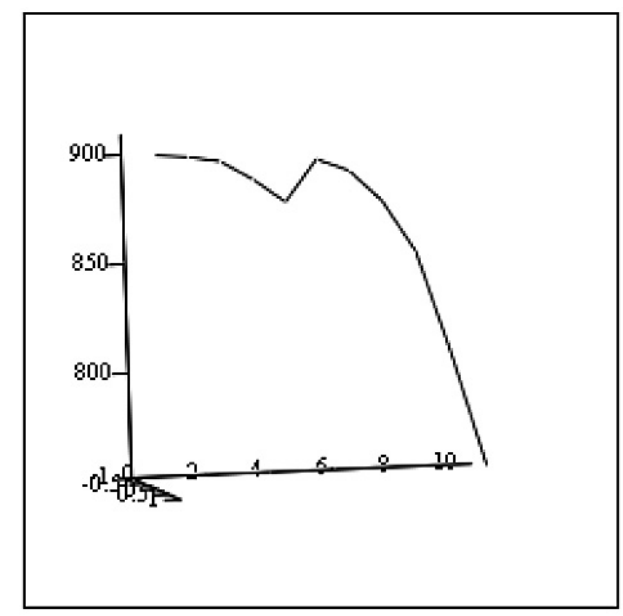

povneyav

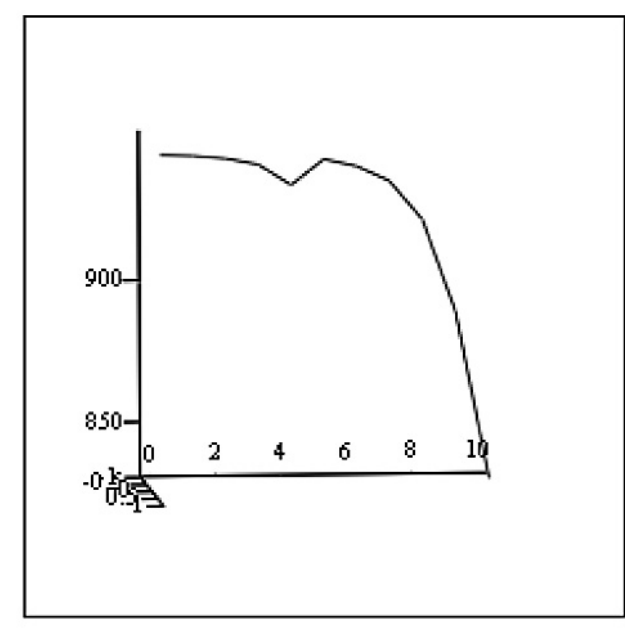

smeyav

Fig. 4. Graph of the strip temperature through passages obtained by calculation using implicit VOC (respectively, surface temperature and the central part of roll)

to $915{ }^{\circ} \mathrm{C}$. Sides contacting only with the environment, are cooled to a temperature of $938^{\circ} \mathrm{C}$.

Theoretically, we assume that, after five passages in the cage strip is cut to length, cooled, and then reheated to a temperature of $950{ }^{\circ} \mathrm{C}$. Further, it was rolled in the group stands for 6 passages, i.e. 6 to 11 cages. The results of the cross-section of roll cage after 11 passages are shown in Figure 3.

According to the theory of metal forming to obtain the desired homogeneous structure necessary to achieve a uniform temperature over the cross section of the rod. As seen in Figure 3, this condition is satisfied, although I realize this condition is almost impossible. The resulting calculation of the implicit local-dimensional pattern of roll temperature of different parts of the passage shown in Figure 4.
Studies show that the structure of the center and surface of the rod characterized by, caused by the temperature difference of about $50{ }^{\circ} \mathrm{C}$ and heating the strip, the condition of up to $950{ }^{\circ} \mathrm{C}$ at the inlet of first and second stand, i.e. temperature curve accurately describe the process of deformation of the metal rod.

\section{CONCLUSION}

Designed algorithm for the numerical simulation of the temperature problem provides a three-dimensional model of the temperature field bands in the deformation in multistand mill, as well as conduct experiments by changing these or other factors af- 
fecting the strength and ductility of a deformable metal, including the shape of the calibration without the need for costly full-scale experiments.Checking the accuracy of the calculation and comparative analysis of temperature fields rolling enables the use of the algorithm locally one-dimensional schemes in the profiled rolling.

\section{Acknowledgements}

This research was financially supported by the National Science Foundation in Kazakhstan.

\section{REFERENCES}

1. Dubinsky F.S., Sosedkova M.A., 2007. Methods of designing temperature regimes of hot bar rolling: $\mathrm{A}$ tutorial textbook. Chelyabinsk: Pub. SUSU, 18 p.

2. Ryndin E.A. 2003. Methods for solving problems of mathematical physics. Textbook. Taganro: Publishing - TRTU, $119 \mathrm{p}$.

3. Samarskyi A.A. 1989. The theory of difference schemes. Third ed., Moscow.

4. Sosedkova M.A., Dubinsky F.S., Dukmasov V.G., Vydrin A.V., 2010. Simulation of thermal processes in order to improve technology of bar rolling. Vestnik of SUSU. A series of "Metallurgy", 15(34), 71-75. 